\title{
Cardamom Ingestion as a Possible Cause of Hematuria
}

\author{
Pietro Ferrara • Iliana Bersani • Giorgia Bottaro • \\ Ottavio Vitelli • Antonio Gatto • Francesca del Bufalo • \\ Pio Liberatore • Lorenza Romani • Achille Stabile
}

Received: 30 December 2010 / Accepted: 2 May 2011 /Published online: 10 June 2011

(C) Dr. K C Chaudhuri Foundation 2011

Sir,

Hematuria in childhood is a common clinical entity which needs an extensive evaluation.

A 5-year-old girl admitted to our Pediatric Department because of isolated hematuria without any flank discomfort. The patient denied having fever, infections or any trauma in previous months. There was no family history of urinary stones or bleeding diathesis. The patient took no medication. Her physical examination was unremarkable. The child was afebrile and normotensive. Initial laboratory evaluation revealed an hemoglobin concentration of $12.5 \mathrm{~g} / \mathrm{dL}$, platelet count of $370.000 / \mathrm{mm}^{3}$, white blood cells count of $8.370 / \mathrm{mm}^{3}$, and creatinine level of $0.7 \mathrm{mg} / \mathrm{dL}$. An urinalysis revealed 88 to 100 red blood cells (RBCs) per hpf, more than 25 dysmorphic RBCs, more than $5 \%$ of acanthocytes and no urinary casts or crystals. Coagulation profile, cistatin C, complement and serum IgA were negative. Twenty four-hour urinalysis revealed normal values of protein and albumin, sodium potassium and calcium. Renal and bladder ultrasonography showed normal appearing of kidneys and bladder. Hematuria spontaneously disappeared within 1 day.

A new analysis of the patient's history demonstrated the intake of cardamom ice cream during dinner before admission. After discharge, a cardamom-free diet was prescribed and laboratoristic follow up was performed. Urinary sticks, daily performed during the first 2 wks after discharge and then wkly for the following months, always resulted negative. Urinalysis carried out at 1, 3 and 6 months after hospitaliza-

P. Ferrara $(\bowtie) \cdot$ I. Bersani $\cdot$ A. Gatto $\cdot$ P. Liberatore $\cdot$ A. Stabile

Department of Pediatric Sciences,

"A. Gemelli" University Hospital,

L.go Gemelli, 8,

00168 Rome, Italy

e-mail: pferrara@rm.unicatt.it

G. Bottaro $\cdot$ O. Vitelli $\cdot$ F. del Bufalo $\cdot$ L. Romani

Campus Bio-Medico University,

Rome, Italy tion, did not show further episodes of hematuria and the child didn't show other clinical signs or symptoms.

Isolated hematuria among children often needs extensive evaluation, since differential diagnosis contemplates a large spectrum of etiologic factors including infections, stones, trauma, cancer, foods, drugs and other substances $[1,2]$.

It has recently been demonstrated that cardamom (Elettaria cardamomum), a highly aromatic spice, is able to inhibit platelet aggregation, blocking the calcium transport across platelet membranes. Moreover cardamom compete with agonists for interaction with glycoproteins IIb-IIIa, not allowing the adherence of fibrinogen and it also interferes with the inhibition of adenylate cyclase activity and intraplatelet cAMP levels [3]. Furthermore, it has been highlighted that other spices and herbals are associated with an increased risk of bleeding by inhibiting platelet function [4].

Considering these findings, it is reasonable to hypothesize that an excessive intake of cardamom may represent a risk factor for the development of hemorrhagic disorders. So physicians should ensure that patients receiving anticoagulant and antiplatelet medications, do not simultaneously assume cardamom. Our case would represent the first report of cardamom ingestion as a possible cause of hematuria.

Further trials are required to investigate about safety of cardamom and other spices, in order to avoid the development of possible side effects.

\section{References}

1. Cohen RA, Brown RS. Clinical practice. Microscopic hematuria. N Engl J Med. 2003;348:2330-8.

2. Phadke KD, Vijayakumar M, Sharma J, et al. Consensus statement on evaluation of hematuria. Indian Pediatr. 2006;43: 965-73.

3. Suneetha WJ, Krishnakantha TP. Cardamom extract as inhibitor of human platelet aggregation. Phytother Res. 2005;19:437-40.

4. Ciocon JO, Ciocon DG, Galindo DJ. Dietary supplements in primary care. Botanicals can affect surgical outcomes and follow up. Geriatrics. 2004;59:20-2. 